\title{
Cloud Type and Top Height Estimation for Tropical Upper-Tropospheric Clouds Using GMS-5 Split-Window Measurements Combined with Cloud Radar Measurements
}

\author{
Atsushi Hamada ${ }^{1}$, Noriyuki Nishi ${ }^{1}$, Suginori Iwasaki², Yuichi Ohno ${ }^{3}$, \\ Hiroshi Kumagai ${ }^{3}$, and Hajime Okamoto ${ }^{4}$ \\ ${ }^{1}$ Division of Earth and Planetary Sciences, Graduate School of Science, Kyoto University, Kyoto, Japan \\ ${ }^{2}$ Department of Earth and Ocean Sciences, National Defense Academy, Yokosuka, Japan \\ ${ }^{3}$ National Institute of Information and Communications Technology, Tokyo, Japan \\ ${ }^{4}$ Center for Atmospheric and Oceanic Studies, Graduate School of Science, Tohoku University, \\ Sendai, Japan
}

\begin{abstract}
Cloud types of tropical upper-tropospheric stratiform clouds (UTSCs) were estimated using split-window brightness temperatures $\left(\mathrm{T}_{\mathrm{B}}\right)$ measured by a geostationary satellite. For non-precipitating high clouds, cloudtop heights were estimated. Observation-based estimation tables in terms of $10.8 \mu \mathrm{m} \mathrm{T}_{\mathrm{B}}\left(\mathrm{T}_{11}\right)$ and the difference between $\mathrm{T}_{11}$ and $12 \mu \mathrm{m} \mathrm{T}_{\mathrm{B}}\left(\Delta \mathrm{T}=\mathrm{T}_{11}-\mathrm{T}_{12}\right)$ were presented using ship-borne cloud radar measurements conducted during three months in the tropical warm-pool region. After defining the cloud types and cloud-top height using radar measurements, their detectabilities were shown as the function of $\mathrm{T}_{11}$ and $\Delta \mathrm{T}$. The detectability of non-precipitating UTSCs is higher in regions with $\mathrm{T}_{11}$ between 220 and $275 \mathrm{~K}$ and higher $\Delta \mathrm{T}$. Surface precipitation is more detectable in regions with low $T_{11}$ and small $\Delta \mathrm{T}$. The estimated cloud-top height of nonprecipitating UTSCs tends to rise with decreasing $\mathrm{T}_{11}$ and increasing $\Delta \mathrm{T}$. The variation in the cloud-top estimates with $\Delta \mathrm{T}$ reached a few kilometers at $\mathrm{T}_{11}$ of $\sim 250 \mathrm{~K}$.
\end{abstract}

\section{Introduction}

Tropical deep convective activity is known to be accompanied by large stratiform clouds extending in the upper troposphere (e.g., Houze 1997). These stratiform clouds (hereafter referred to as upper-tropospheric stratiform clouds; UTSCs) generally consist of nimbostratus with precipitation and cirriform clouds without precipitation (non-precipitating UTSCs).

Non-precipitating UTSCs often extend up to more than a thousand kilometers, and play a key role in maintaining the water vapor content in the upper troposphere. To clarify how tropical cloud systems influence the moisture content in the tropical troposphere, knowledge about the spatial and temporal evolution of the vertical location and ice content of the non-precipitating UTSCs is required. The non-precipitating UTSCs also play an important role in the Earth's radiative budget. They heat the atmosphere by absorbing terrestrial radiation, and simultaneously cool it by reflecting solar radiation. The degree to which the atmosphere is heated or cooled depends on the UTSCs' geometrical and radiative characteristics, such as the cloud-top height and optical thickness (e.g., Hartmann 2001). However,

Corresponding author: Atsushi Hamada, Division of Earth and Planetary Sciences, Graduate School of Science, Kyoto University, Kitashirakawa Oiwake-cho, Sakyo, Kyoto 606-8502, Japan. E-mail: hamada@kugi.kyoto-u.ac.jp. @2008, the Meteorological Society of Japan. global distribution of the cloud-top height and optical thickness of the non-precipitating UTSCs is estimated only roughly.

Infrared brightness temperatures $\left(T_{B}\right)$ measured by geostationary satellites are often used to detect the convective activity that generates the UTSCs (e.g., Udelhofen and Hartmann 1995). However, a threshold adopted in previous studies uses only a single infrared $\mathrm{T}_{\mathrm{B}}$, and has difficulty discriminating between precipitating UTSCs and optically thick non-precipitating UTSCs. Geostationary satellites are most effective for observing large, long-lived tropical cloud systems. In the tropics, diurnal cloud variation dominates (e.g., Chen and Houze 1997), and the non-precipitating UTSCs often persist for up to a day. Therefore, to describe the temporal evolution of the UTSCs, a data and analysis method applicable during both daytime and nighttime, is preferred.

The split-window method (Inoue 1985, 1987) using two $T_{B}$ measurements at infrared window wavelengths, e.g., $10.8\left(\mathrm{~T}_{11}\right)$ and $12 \mu \mathrm{m}\left(\mathrm{T}_{12}\right)$, can be used during both daytime and nighttime, and it is applicable to recent geostationary satellites. In a simplified radiative transfer model of the ground surface and a single-layer "cloud" without geometrical thickness (hereafter referred to as the simplistic model), the height and optical thickness of the cloud is expressed in terms of $T_{11}$ and $\Delta \mathrm{T}=\mathrm{T}_{11}-\mathrm{T}_{12}$ (e.g., Cooper et al. 2003). Using this fact, extensive algorithms have been developed to classify clouds (e.g., Luo et al. 2002) and estimate the cloud-top height and optical thickness (e.g., Inoue 1985), using split-window $T_{B}$ measurements. However, the simplistic model is highly sensitive to other parameters such as the effective radius and shape of cloud ice (Stephens and Kummerow 2007). Validation using observational data is required.

Validation of the split-window methods using in-situ observational data has been limited to small areas and/or short periods because of the difficulty in observing the non-precipitating UTSCs. Recently, datasets have become available with observations using instruments, such as cloud radar and lidar, which can probe the internal structure of the non-precipitating UTSCs. The purpose of this study is to present an estimation table of the cloud type and cloud-top height using splitwindow $\mathrm{T}_{\mathrm{B}}$ measured by a geostationary satellite. The table is validated using ship-borne cloud radar measurements conducted during three months.

\section{Data and analysis method}

We used two brightness temperature $\left(T_{B}\right)$ measurements at infrared split-window wavelengths observed by the fifth Geosynchronous Meteorological Satellite 
(GMS-5). Response functions for two channels are centered at $10.8\left(\mathrm{~T}_{11}\right)$ and $12 \mu \mathrm{m}\left(\mathrm{T}_{12}\right)$, respectively. The horizontal resolution of the original data is $\sim 4 \mathrm{~km}$ at nadir $\left(140^{\circ} \mathrm{E}, 0^{\circ} \mathrm{N}\right)$; hourly data on a $0.05^{\circ}$ grid is used in this study. The temperature resolution is $\sim 0.4 \mathrm{~K}$ at 300 $\mathrm{K}$, and it is slightly coarser at lower temperatures, $\sim 1.4$ $\mathrm{K}$ at $210 \mathrm{~K}$. To define the cloud types and estimate the cloud-top height, radar reflectivity $(Z)$ and Doppler velocity $\left(\mathrm{V}_{\mathrm{D}}\right)$ measurements obtained from the millimeter-wave (wavelength $3.16 \mathrm{~mm}$ ) cloud radar developed by National Institute of Information and Communications Technology (NICT) were used. This radar was installed on Mirai, which is a research vessel of the Japan Marine Science and Technology Center (JAMSTEC), and made vertical observations in the tropical warm-pool region from September to December 2001. In this study, we used data between $90^{\circ} \mathrm{E}-140^{\circ} \mathrm{E}$ and $15^{\circ} \mathrm{S}-15^{\circ} \mathrm{N}$. The vertical and temporal resolutions were $82.5 \mathrm{~m}$ and $10 \mathrm{~s}$, respectively.

We obtained 1317 samples with paired cloud radar and GMS-5 measurements during the analysis period, as described below. The vertical profiles of $Z$ and $V_{D}$ were averaged within 2 minutes centered at the time when GMS-5 observed the location of the vessel. Corresponding values of $T_{11}$ and $\Delta \mathrm{T}$ were obtained by linear spatial interpolation, using four grids around the vessel. For each cloud radar profile, cloud layers were defined as at least three consecutive vertical bins, where the radar signal was $0.1 \mathrm{~dB}$ above the noise level (Okamoto et al. 2003). The precipitation flag was set when the radar profile had any vertical bin with $Z>-16 \mathrm{dBZ}$ (Wang and Geerts 2003) and $V_{D} \leq-1 \mathrm{~m} \mathrm{~s}^{-1}$ below $3 \mathrm{~km}$.

The samples were classified into five categories (hereafter referred to as cloud types) using cloud layer and precipitation flag information; 1) Non-precipitating high clouds that has echo top height $\left(z_{\mathrm{T}}\right)$ higher than 7 $\mathrm{km}$ (H-NR-type), 2) Non-precipitating low clouds that has echo top height lower than $7 \mathrm{~km}$ (L-NR-type), 3) Surface precipitating clouds with non-precipitating clouds $3 \mathrm{~km}$ higher than them (M-type), 4) Surface precipitating clouds with no accompanying nonprecipitating clouds (R-type), and 5) Clear sky (C-type). Note that some of R-type samples may be M-type, because the cloud radar signal is heavily attenuated by precipitation particles. There were 508, 27, 42, 143, and 597 samples of H-NR, L-NR, M-, R-, and C-type, respectively.

We defined the occurrence rate of each cloud type in $\mathrm{T}_{11}-\Delta \mathrm{T}$ space as follows. First, the bivariate probability density function (PDF) in $T_{11}-\Delta T$ space was computed for each cloud type using a two-dimensional Epanechnikov kernel in both the $\mathrm{T}_{11}$ and $\Delta \mathrm{T}$ dimensions (Silverman 1986). Smoothing parameters for PDF estimation were computed by the maximum-likelihood cross-validation technique. The computed smoothing parameters for each dimension were $(2.97,0.39)$, (3.92, $0.62),(9.07,0.43),(4.44,0.39)$, and $(2.87 \mathrm{~K}, 0.27 \mathrm{~K})$ for $\mathrm{H}-$ NR, L-NR, M-, R-, and C-type, respectively. Next, each PDF was multiplied by the ratio of the number of corresponding samples to that of all samples. Finally, the occurrence rate of each cloud type in $T_{11}-\Delta \mathrm{T}$ space was calculated as the ratio of the corresponding PDF to the sum of PDFs for all the cloud types.

For estimating the cloud-top height using GMS-5 split-window $T_{B}$ measurements, we used the value of $z_{T}$ as the index of the cloud-top height, which was estimated by nonparametric regression of $z_{\mathrm{T}}$ over $T_{11}$ and $\Delta \mathrm{T}$. We adopted the Nadaraya-Watson estimator (Scott 1992) with the same kernel as used in PDF estimation. The smoothing parameter was computed again by the least-square cross-validation technique, and was $(6.98 \mathrm{~K}$, $0.39 \mathrm{~K})$. In this study, we show the results only for $\mathrm{H}$ -
NR-type samples, because the value of $z_{\mathrm{T}}$ could not be defined by ground-based cloud radar observations under precipitating conditions.

\section{Results}

\subsection{Distribution and occurrence rate of each cloud type in $T_{11}-\Delta T$ space}

Figure 1 shows the distribution of all samples in $\mathrm{T}_{11}-\Delta \mathrm{T}$ space, as well as the common logarithm of the bivariate PDF for H-NR- (solid lines) and R-type (dashed lines) samples. The H-NR-type samples (green) are distributed broadly in the $\mathrm{T}_{11}$ direction. PDF for H-NR-type shows that the center of distribution along the $\Delta \mathrm{T}$ axis varies with $T_{11}$. It peaks near $3 \mathrm{~K}$ at around $\mathrm{T}_{11}=270 \mathrm{~K}$, and drops with both increasing and decreasing $\mathrm{T}_{11}$. The R-type samples (red) are also distributed broadly in the $\mathrm{T}_{11}$ direction. However, the distribution along the $\Delta \mathrm{T}$ axis differs substantially from that of the H-NR-type. The center of R-type distribution along the $\Delta \mathrm{T}$ axis tends to remain at $\Delta \mathrm{T}$ lower than $1 \mathrm{~K}$ in the region where $\mathrm{T}<270 \mathrm{~K}$. The M-type samples (yellow) tend to have a larger $\Delta \mathrm{T}$ than the R-type samples. The centers of distribution along the $\Delta \mathrm{T}$ axis are at $\sim 2.5 \mathrm{~K}$ between 230 and $260 \mathrm{~K}$, and $\sim 1.5 \mathrm{~K}$ elsewhere (not shown). The distributions of both C- (blue) and L-NR-type (light blue) samples concentrate in high- $\mathrm{T}_{11}$ regions, with minor exceptions.

The observed difference in the distributions of the $\mathrm{H}$ NR- and R-type samples indicates the detectability of the non-precipitating UTSCs using GMS-5 split-window $\mathrm{T}_{\mathrm{B}}$. We calculated the sum of H-NR- and M-type occurrence rates for examining the occurrence rate of the non-precipitating UTSCs, regardless of the precipitation from the underneath cloud. The results are shown in Fig. 2. The occurrence rate basically increases with $\Delta \mathrm{T}$ when $\mathrm{T}_{11}<270 \mathrm{~K}$. The highest value, which is over $80 \%$, is found in the region with $\mathrm{T}_{11}$ between 220 and $270 \mathrm{~K}$ and large $\Delta T$. Since the H-NR-type samples tend to show a high value of $\Delta \mathrm{T}$ in this region, as mentioned above (Fig. 1), this demonstrates that the high value of $\Delta \mathrm{T}$ can effectively detect the non-precipitating UTSCs. The occurrence rate falls in the region with $\mathrm{T}>280 \mathrm{~K}$, mainly due to the higher number of samples belonging to the Cor the L-NR-type. It is noteworthy that isopleths between 50 and 70\% in Fig. 2 tend to rise with decreasing $\mathrm{T}_{11}$ when $\mathrm{T}_{11}<265 \mathrm{~K}$. Constant values of $\Delta \mathrm{T}$ with respect to $T_{11}$ have been used as a threshold value to detect cirrus clouds by the split-window $\mathrm{T}_{\mathrm{B}}$ (e.g., Luo et al. 2002). However, the observation-based results (Fig. 2 ) indicate that threshold values of $\Delta \mathrm{T}$ for cloud classification are dependent on $\mathrm{T}_{11}$ rather than being constant.

Next we examined the detectability of surface precipitation using GMS-5 split-window $\mathrm{T}_{\mathrm{B}}$ measurements, by showing the sum of R-and M-type occurrence rates (Fig. 3). Note that the sum of the values shown in Figs. 2 and 3 exceeds 100\% in some regions. Higher values are found in regions with lower $T_{11}$ and smaller $\Delta \mathrm{T}$. In the region with $T_{11}>220 \mathrm{~K}$, the occurrence rate increases with decreasing $\Delta \mathrm{T}$, indicating that split-window $\mathrm{T}_{\mathrm{B}}$ can estimate tropical rainfall more effectively than a single infrared channel method such as the GOES Precipitation Index (GPI; Arkin and Meisner 1987).

It is noteworthy that the occurrence rate of nonprecipitating UTSCs is still up to $\sim 50 \%$ in the region with very low $T_{11}$ and small $\Delta \mathrm{T}$, since the single-channel threshold of $\mathrm{T}_{11}$ around $210 \mathrm{~K}$ has been believed to detect the precipitating core of tropical deep convective activity (e.g., Chen and Houze 1997). We examined the time-height cross section of $Z$ for all H-NR- and M-type samples located in the region where an occurrence rate 


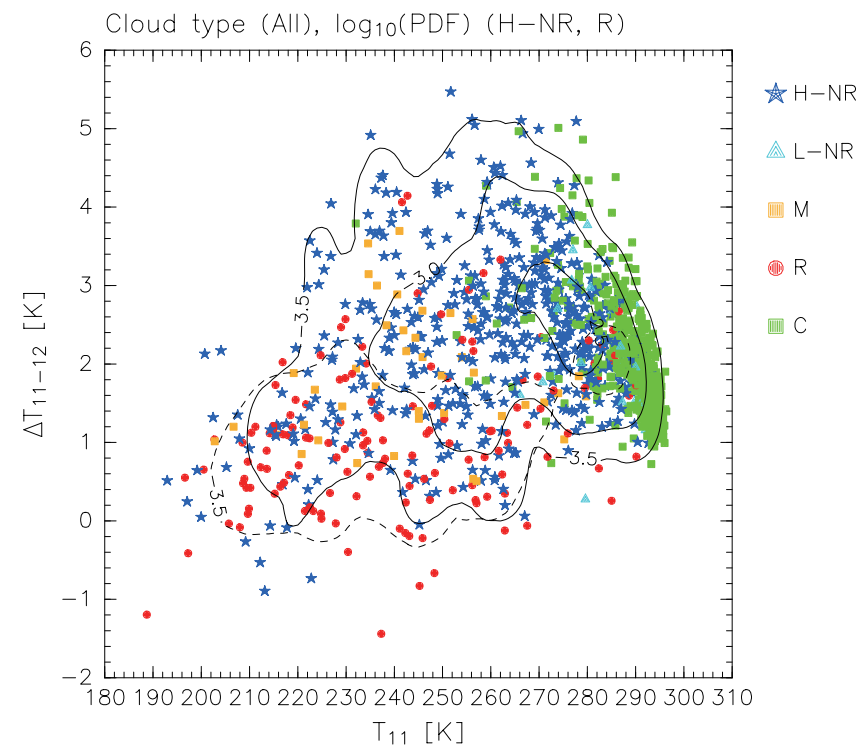

Fig. 1. Distribution of samples of all cloud types defined by cloud radar profiles. Ordinate and abscissa indicate $\mathrm{T}_{11}$ and $\Delta \mathrm{T}$, respectively. H-NR, L-NR, M-, R-, and C-type samples are indicated by green, light blue, yellow, red, and blue marks, respectively. Solid and dashed lines indicate the sum of bivariate PDFs for H-NR- and R-type samples, respectively. The PDF is shown as a common logarithm and the contour interval is 0.5 from -3.5 .

of surface precipitation higher than 60\%. As a result, more than $7-\mathrm{km}$ thick stratiform cloud layers were observed in about two-thirds of all samples (not shown). It is believed that these dense cloud layers originated from nimbostratus with deep convective activity in the tropics. Therefore, even where the occurrence rate of surface precipitation is $\sim 60 \%$, it is very likely that convective activity exists close to GMS-5 observation point.

\subsection{Cloud-top height estimation for non-precipitating UTSCS}

In the simplistic model, the relationship between $\mathrm{T}_{11}$ and $\Delta \mathrm{T}$ when the height and effective radius of the "cloud" remains constant shows an upward arch in $\mathrm{T}_{11}$ $\Delta \mathrm{T}$ space (e.g., Cooper et al. 2003). The arched distribution of the H-NR-type samples in $\mathrm{T}_{11}-\Delta \mathrm{T}$ space (Fig. 1) is reminiscent of the curves of $T_{11}$ versus $\Delta T$ for the cloudtop heights expected from this model. Figure 4 shows the result of cloud-top height estimation for the H-NRtype samples, obtained by regressing $z_{\mathrm{T}}$ over $\mathrm{T}_{11}$ and $\Delta \mathrm{T}$. The estimated $z_{\mathrm{T}}$ (solid lines) generally increases with decreasing $\mathrm{T}_{11}$ and increasing $\Delta \mathrm{T}$. Isopleths mostly tend to tilt rightward from the vertical, except in the region with high $\mathrm{T}_{11}$ and small $\Delta \mathrm{T}$. The variation in the cloudtop estimates varies significantly with $\Delta \mathrm{T}$, and reaches a few kilometers at $T_{11}$ of $\sim 250 \mathrm{~K}$. The standard deviations of $z_{\mathrm{T}}$ (dashed lines) tend to be small, as the $z_{\mathrm{T}}$ estimate increases. They peak at most $\sim 2.0 \mathrm{~km}$ when the estimated $z_{\mathrm{T}}$ is higher than $12 \mathrm{~km}$. The above results demonstrate that GMS-5 split-window $\mathrm{T}_{\mathrm{B}}$ effectively estimates the cloud-top height of the non-precipitating UTSCs.

It is interesting to compare the results shown in Fig. 4 with the following two estimation methods based on the simplistic model. The first is a single infrared channel method, where the value of $T_{11}$ is considered to be equal to the cloud-top temperature. This method may

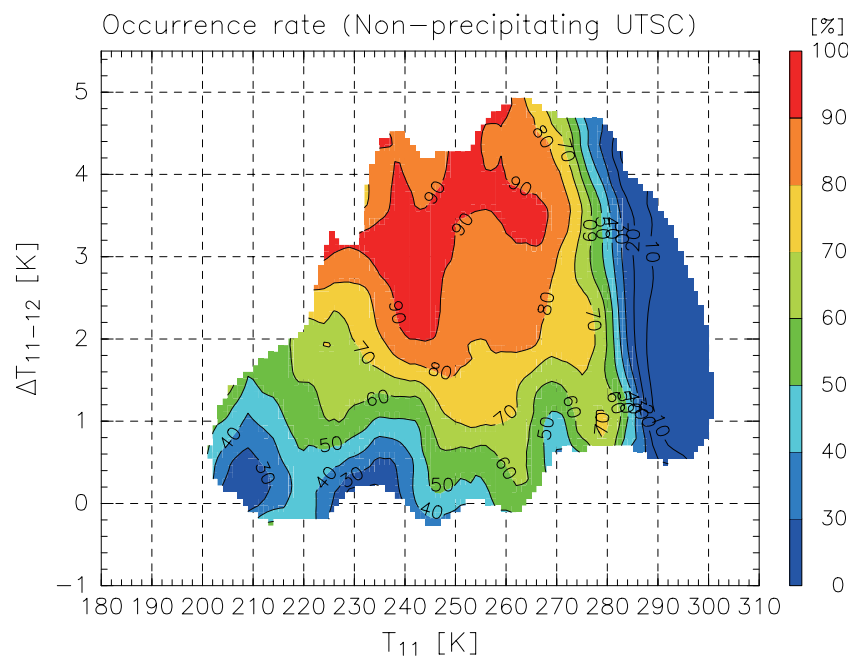

Fig. 2. Occurrence rates of non-precipitating UTSCs as the sum of H-NR- and M-type occurrence rates in $\mathrm{T}_{11}-\Delta \mathrm{T}$ space (see text for details). The contour interval is $10 \%$. Contours and shadings are omitted where the $\log _{10}(\mathrm{PDF})$ in Fig. 1 is less than -3.3 .

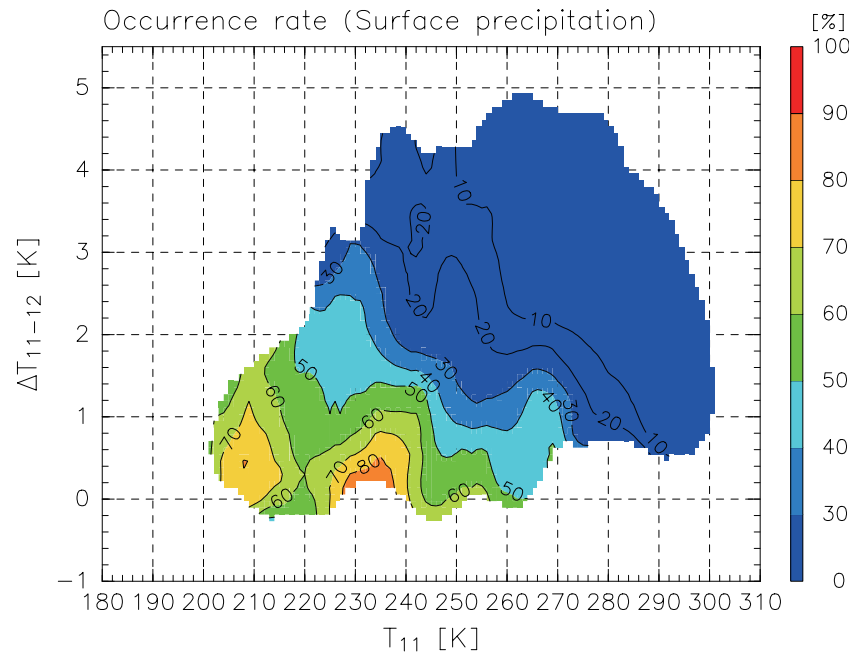

Fig. 3. Same as Fig. 2, but for sufrace precipitation as the sum of R-and M-type occurrence rates.

significantly underestimate the cloud-top height, particularly for clouds with $\mathrm{T}_{11}$ of $\sim 250 \mathrm{~K}$ and large $\Delta \mathrm{T}$. The second is a two-channel method using split-window measurements. Curves of $\mathrm{T}_{11}$ versus $\Delta \mathrm{T}$ for $\mathrm{z}_{\mathrm{T}}$ are largely consistent with those for cloud-top heights expected from the simplistic model, while the estimated $z_{\mathrm{T}}$ at around $\Delta T=0$ shows a notable feature. In the simplistic model, zero $\Delta \mathrm{T}$ means that the cloud has infinitely large optical thickness, and is located at a height where the temperature just equals $\mathrm{T}_{11}$. However, even in regions where $T_{11}$ is low enough to neglect the water vapor effect, $z_{\mathrm{T}}$ estimated using cloud radar measurements is still higher than expected from the mean temperature profile computed from the ERA-40 objective analysis data over the analysis period (not shown). For example, a temperature of $220 \mathrm{~K}$ corresponds to $\sim 12.5 \mathrm{~km}$ in the tropics, whereas the estimate of $z_{\mathrm{T}}$ at $\mathrm{T}_{11}=220 \mathrm{~K}$ and $\Delta \mathrm{T}$ $=0 \mathrm{~K}$ is $\sim 1.5 \mathrm{~km}$ higher. This discrepancy is of interest for future studies. 


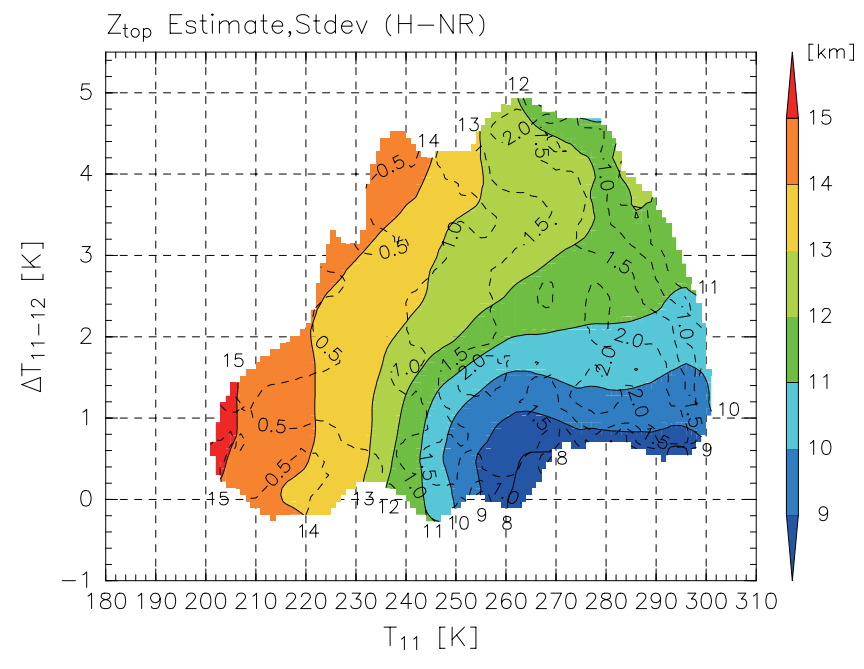

Fig. 4. Estimates (shading) and standard deviations (dashed contours) of $z_{\mathrm{T}}$ for H-NR-type samples obtained by regression of $z_{T}$ over $T_{11}$ and $\Delta T$ (see text for details). The contour intervals are $1 \mathrm{~km}$ and $0.5 \mathrm{~km}$ for the estimates and standard deviations, respectively. No contours are shown where the $\log _{10}(\mathrm{PDF})$ in Fig. 1 is less than -3.3 .

\section{Concluding remarks}

An estimation table of types of tropical uppertropospheric clouds determined by GMS-5 split-window brightness temperature $\left(T_{B}\right)$ measurements was shown. For non-precipitating high clouds, the cloud-top height was also estimated using split-window $\mathrm{T}_{\mathrm{B}}$. The estimation table shown here is validated using ship-borne cloud radar measurements conducted during three months.

Distribution of cloud types defined from cloud radar measurements is shown in $\mathrm{T}_{11}-\Delta \mathrm{T}$ space. After calculating the bivariate PDF for each cloud type, the occurrence rate in $T_{11}-\Delta \mathrm{T}$ space was determined for nonprecipitating UTSCs and surface precipitation. Samples of non-precipitating UTSCs (H-NR-type) are broadly distributed in $T_{11}-\Delta \mathrm{T}$ space, while samples of precipitating clouds (R-type) occur in the region where $\Delta \mathrm{T}$ is small. Distributions of H-NR- and R-type samples differ particularly in the region with $T_{11}$ between 220 and $275 \mathrm{~K}$, resulting in a high occurrence rate of non-precipitating UTSCs in this region. Occurrence rates of nonprecipitating UTSC generally tend to increase with increasing $\Delta \mathrm{T}$. The inclination of isopleths in the region with $\mathrm{T}_{11}<265 \mathrm{~K}$ suggests that the threshold value of $\Delta \mathrm{T}$ for cloud classification should be dependent on $T_{11}$ rather than being constant.

Cloud-top height estimates of the H-NR-type samples generally rise with decreasing $\mathrm{T}_{11}$ and increasing $\Delta \mathrm{T}$. The variation along $\Delta \mathrm{T}$ reaches a few kilometers at $\mathrm{T}_{11}$ of $\sim 250 \mathrm{~K}$, indicating that information about $\Delta \mathrm{T}$ is important for the estimation of cloud-top heights of the non-precipitating UTSCs. The shape of isopleths for estimated $z_{\mathrm{T}}$ is largely consistent with those for cloud-top height expected from the simplistic model. Observationbased quantitative results were shown in the present study.

Spaceborne cloud radar measurements can estimate the cloud-top height, regardless of precipitating or non- precipitating conditions. We are currently undertaking an analysis using cloud radar measurements from the CloudSat satellite. Using this radar measurements, the analysis method employed in the present study can be applied easily to data from other geostationary satellites that perform split-window $T_{B}$ observations. Records of more than one year would clarify the regional, seasonal, and diurnal variation of UTSC properties.

\section{Acknowledgments}

The authors thank Dr. T. Satomura for helpful discussions. The figures were produced by GFD-Dennou Library.

\section{References}

Arkin, P. A., and B. N. Meisner, 1987: The relationship between large-scale convective rainfall and cold cloud over the western hemisphere during 198284. Mon. Wea. Rev., 115, 51-74.

Chen, S. S., and R. A. Houze Jr., 1997: Diurnal variation and life-cycle of deep convective systems over the tropical Pacific warm pool. Quart. J. Roy. Meteor. Soc., 123, 357-388.

Cooper, S. J., T. S. L'Ecuyer, and G. L. Stephens, 2003: The impact of explicit cloud boundary information on ice cloud microphysical property retrievals from infrared radiances. J. Geophys. Res., 108, doi: 10.1029/2002JD002611.

Houze, R. A., Jr., 1997: Stratiform precipitation in regions of convection: A meteorological paradox? Bull. Amer. Meteor. Soc., 78, 2179-2196.

Inoue, T., 1985: On the temperature and effective emissivity determination of semi-transparent cirrus clouds by bi-spectral measurements in the $10 \mu \mathrm{m}$ window region. J. Meteor. Soc. Japan, 63, 88-99.

Inoue, T., 1987: A cloud type classification with NOAA 7 split-window measurements. J. Geophys. Res., 92, 3991-4000.

Luo, Z., W. B. Rossow, T. Inoue, and C. J. Stubenrauch, 2002: Did the eruption of the Mt. Pinatubo volcano affect cirrus properties? J. Climate, 15, 2806-2820.

Okamoto, H., S. Iwasaki, M. Yasui, H. Horie, H. Kuroiwa, and H. Kumagai, 2003: An algorithm for retrieval of cloud microphysics using $95-\mathrm{GHz}$ cloud radar and lidar. J. Geophys. Res., 108, doi:10.1029/2001 JD001225.

Scott, D. W., 1992: Multivariate Density Estimation: Theory, Practice, and Visualization. Wiley-Interscience, $317 \mathrm{pp}$.

Silverman, B. W., 1986: Density Estimation for Statistics and Data Analysis. Chapman \& Hall, 176 pp.

Stephens, G. L., and C. D. Kummerow, 2007: The remote sensing of clouds and precipitation from space: A review. J. Atmos. Sci., 64, 3742-3765.

Udelhofen, P. M., and D. Hartmann, 1995: Influence of tropical cloud systems on the relative humidity in the upper troposphere. J. Geophys. Res., 100, 74237440 .

Wang, J., and B. Geerts, 2003: Identifying drizzle within marine stratus with W-band radar reflectivity. Atmos. Res., 69, 1-27.

Manuscript received 3 April 2008, accepted 11 June 2008 SOLA: http://www.jstage.jst.go.jp/browse/sola/ 